\title{
Left Atrial Appendage Perforation by the Watchman Device: Considerations for Emergency Repair
}

\author{
Saqib Masroor ${ }^{1}$, Qiong Qiu ${ }^{1}$, Mohamad Alghothani ${ }^{2}$, rajesh Gupta ${ }^{2}$, and george moukarbel ${ }^{1}$ \\ ${ }^{1}$ University of Toledo Medical Center \\ ${ }^{2}$ University of Toledo - Health Science Campus
}

August 23, 2020

\begin{abstract}
Percutaneous occlusion of the left atrial appendage is increasingly being used for stroke prevention in patients with nonvalvular atrial fibrillation $(\mathrm{AF})$ at high risk of complications from long term anticoagulation. We describe a case of left atrial appendage perforation after Watchman device deployment requiring emergency repair of the left atrium using sternotomy and cardiopulmonary bypass. Technical considerations for surgical decision making are discussed, in hemodynamically unstable patients as well as those at high risk for embolization.
\end{abstract}

\section{Introduction}

Left atrial appendage (LAA) occlusion with the Watchman device (Boston Scientific, Marlborough, MA) is increasingly being used as an alternative for stroke prevention in patients with non-valvular atrial fibrillation (AF) at high risk of complications from long term anticoagulation (1). Although the procedure has an overall favorable safety profile, it does carry a small risk of serious complications. Compared to the initial PROTECT AF trial, the subsequent PREVAIL trial demonstrated a reduction in the incidence of procedural complications from $8.7 \%$ to $4.2 \%$. In particular, post-procedural pericardial effusions requiring pericardiocentesis or surgery were significantly reduced, with only one reported case of cardiac perforation among 269 patients in the device group (1). Here we report a case of left atrial appendage perforation resulting from Watchman device deployment requiring emergency repair of the left atrium using sternotomy and cardiopulmonary bypass and discuss options for surgical management of this complication. Informed consent was waived by Institutional Review Board.

\section{Case Report}

The patient is an 82-year-old male with a past medical history of coronary artery disease (post stenting), seizure disorder, gastrointestinal (GI) bleeding, paroxysmal AF, CHA2DS2-VASc score of 3, and HASBLED score of 3. Due to his history of GI bleed and fall risk, he underwent LAA occlusion with Watchman device with moderate sedation. Under intracardiac echocardiography (ICE) and fluoroscopic guidance, a $21 \mathrm{~mm}$ Watchman device was deployed. Post-deployment angiography revealed brisk contrast extravasation in the pericardial space. The device was retracted and redeployed at a more proximal position in the ostium of the LAA, and the delivery system was disconnected. The patient developed cardiac tamponade which was confirmed by transthoracic echocardiogram. Emergency pericardiocentesis was performed with the evacuation of $800 \mathrm{~mL}$ of blood. Protamine was administered. Cardiothoracic surgery was emergently consulted. Since the patient continued to require pressor support, he was transferred to the operating room for emergency repair of presumed LAA perforation.

Due to the high risk of embolization of the device and the resulting clot, the plan was to not manipulate LAA until cardiopulmonary bypass was established. Following median sternotomy, the patient's systolic blood 
pressure was $80 \mathrm{~mm} \mathrm{Hg}$, and there was persistent slow bleeding. The patient was immediately heparinized, and cardiopulmonary bypass was initiated after standard cannulation. With the heart empty and beating, we exposed the LAA and identified a large clot adherent to the LAA. Once the clot was removed, the device anchors were seen protruding through the LAA (Figure 1, Video 1) with active bleeding in between the anchors.

Next, antegrade cardioplegic arrest was achieved, and the left atrium was explored through the interatrial groove. The 8-9 $\mathrm{mm}$ septal defect created by the transseptal puncture was repaired with 3-0 monofilament suture. However, the Watchman device was not visible at the ostium It had been pushed into the LAA. The device was delicately pulled out through the left atrium (Figure 2), taking care to unentangle the anchors embedded in the wall of the LAA (Video 2). Upon close examination, there were clots adherent to both the anchors and the nitinol stent cover of the device (Fig 3). Left atrial Cryomaze procedure was then performed using 2-minute ablations encircling the four pulmonary veins as an island. Another lesion was created connecting the left inferior pulmonary vein to the P3 region of the mitral annulus. The LAA was then excluded externally at the base with an epicardial Atriclip (Atricure Inc, Cincinnati, OH). The atriotomy was closed with 3-0 monofilament and cross-clamp removed. After the heart was de-aired, the patient was successfully weaned off of cardiopulmonary bypass. The rest of the procedure and his postoperative course were uneventful. He was discharged on postoperative day 5 in normal sinus rhythm on beta-blockers without antiarrhythmics drugs or anticoagulants.

\section{Comment}

Despite the low reported incidence of post-procedure pericardial effusion and device dislodgement in the randomized trials (1.5\% and $0.74 \%$, respectively in PREVAIL), the real-life complication rate proved to be even better than that seen in trials (2). While surgical repairs of perforation have been reported in the registry, there is no data on what surgical repair was performed. This is the first report of Watchman device associated LAA perforation requiring emergent open surgical repair and discusses the choice of surgical options.

One reported case of LAA perforation was successfully treated with thoracoscopic stapling of the left atrial appendage (3). In this case, the entire device extruded into the pericardium before deployment; therefore, retraction of the delivery system controlled frank hemorrhage by compressing the LAA allowing the surgeons to use a thoracoscopic approach to staple the base of the LAA. The device delivery system was disconnected and withdrawn just as the stapler was fired, thus sealing the two edges of the LAA.

Despite our experience with the thoracoscopic epicardial exclusion of LAA, we chose an open surgical approach for this case for several reasons (4). First, because the watchman device had already been deployed, it could have embolized during the procedure during an off-pump repair. Secondly, the patient was given protamine in the catheterization laboratory in an effort to stop the bleeding, and therefore had an increased likelihood of thrombus formation on and around the device, which could also embolize. Third, a previous study demonstrated that the stapling of the LAA could leave a large stump of LAA, which can be a nidus for future thrombi (5). In contrast, the epicardial clip application results in the complete exclusion of the LAA without leaving any residual stump (6). Finally, because the treatment of AF is important for reducing stroke risk, the Cryomaze procedure was performed at the time of initial surgical repair. The Cyromaze procedure can be performed with a relatively short pump and cross-clamp time and has been reported to have $100 \%$ success in treating paroxysmal AF in contemporary literature (7).

In conclusion, LAA appendage perforation by the Watchman device is an uncommon but serious complication that can be managed with either endovascular, thoracoscopic, or open surgical approach. An individualized approach is needed for each situation based on factors such as the technique of device deployment and the patient's hemodynamic and overall health status.

\section{References}

1. Holmes DR, Kar S, Price KJ, et al. Prospective randomized evaluation of the Watchman left atrial 
appendage closure device in patient with atrial fibrillation versus long-term warfarin therapy. J Am Coll Cardiol 2014;64:1-12Ellis CR, Aznaurov SG, Patel NJ et al. Angiographic efficacy of the Atriclip left atrial appendage exclusion device placed by minimally invasive thoracoscopic approach. J Am Coll Cardiol Electrophysiol 2017;3:1356-65

2. Freeman JV, Varosy P, Price MJ, et al. The NCDR Left Atrial Appendage Occlusion Registry. J Am Coll Cardiol . 2020;75(13):1503-1518. doi:10.1016/j.jacc.2019.12.040

3. Liu J, Liu F-Z, Fang X-H, et al. A unique emergency maneuver and rescue during left atrial appendage occlusion device-related cardiac perforation. J Am Coll Cardiol Electrophysiol 2018:4(5):699-700

4. Smith NE, Joseph J, Morgan J and Masroor S. Initial experience with minimally invasive surgical exclusion of the left atrial appendage with an epicardial clip. Innovations 2017;12(1):28-32

5. Kanderian AS, Gillinov AM, Pettersson CB, Blackstone E and Klein AL. Success of surgical left atrial appendage closure: Assessment by transesophageal echocardiography. J Am Coll Cardiol 2008:52(11):924-9

6. Emmert MY, Puippe G, Baumuller S, et al. Safe, effective and durable epicardial left atrial appendage clip occlusion in patients with atrial fibrillation undergoing cardiac surgery: first long-term results from a prospective device trial. Eur J Cardiothorac Surg 2014;45:126-31

7. Kakuta T, Fukushima S, Minami K, et al. Contemporary outcomes of the concomitant CryoMaze procedure. Interactive Cardiovasc and Thorac Surg 2019:29:28-34

\section{Figure Legends}

Figure 1: The entire Watchman device with anchors (arrows) protruding through the LAA.

Figure 2:The Watchman device delicately pulled out through the left atrium

Figure 3: Extracted Watchman device with visible adherent clot around the anchors (arrow) 


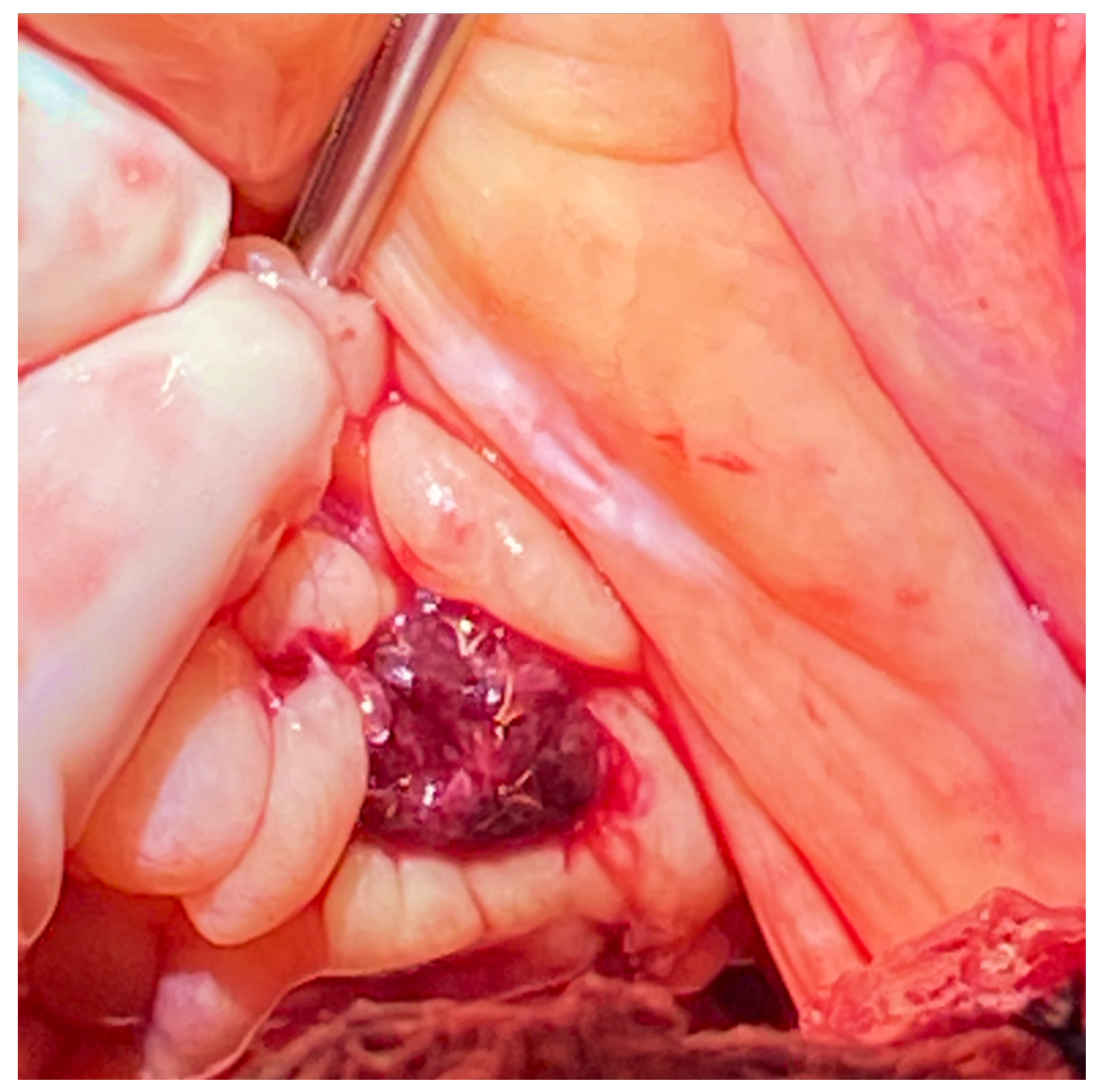




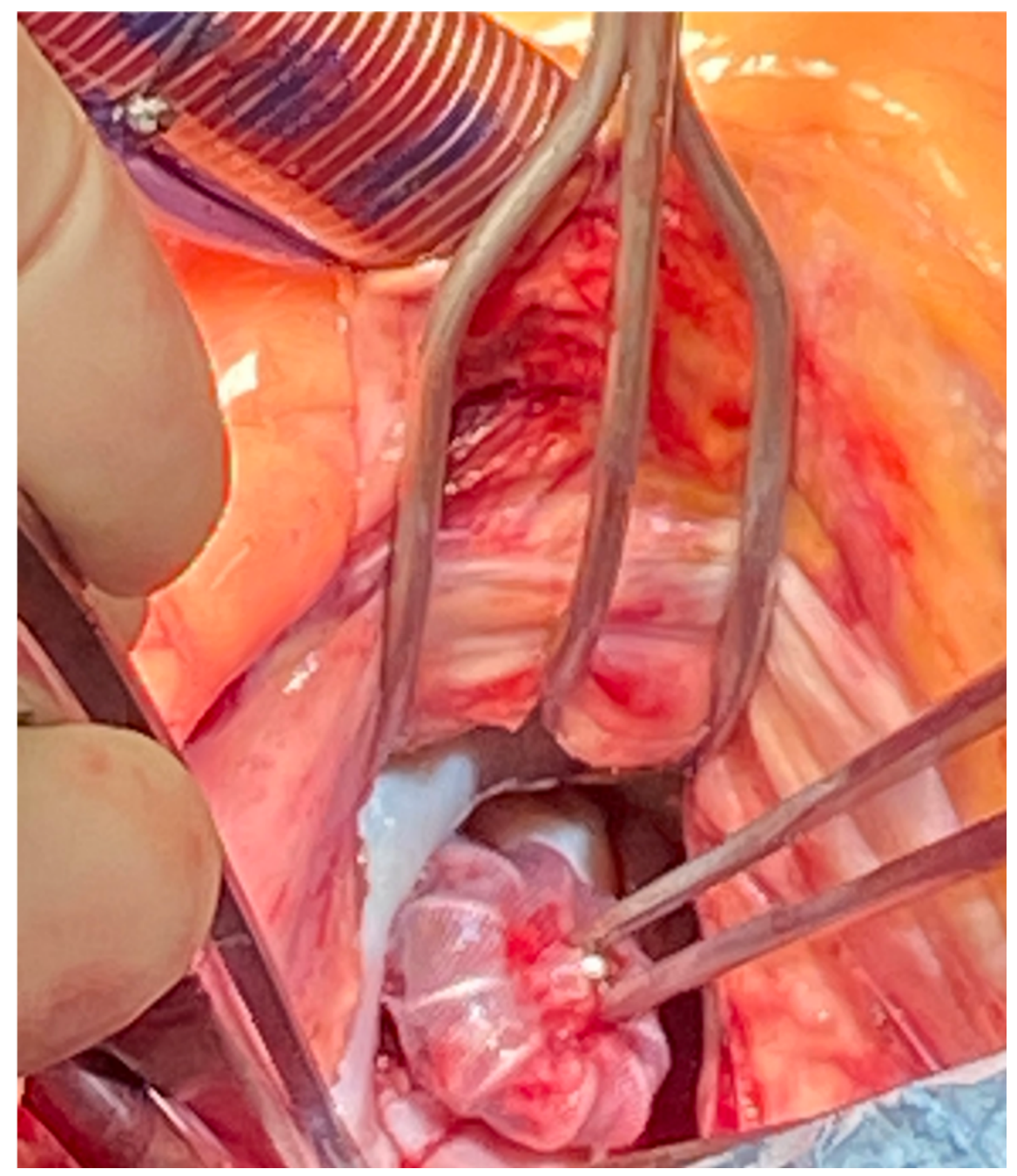




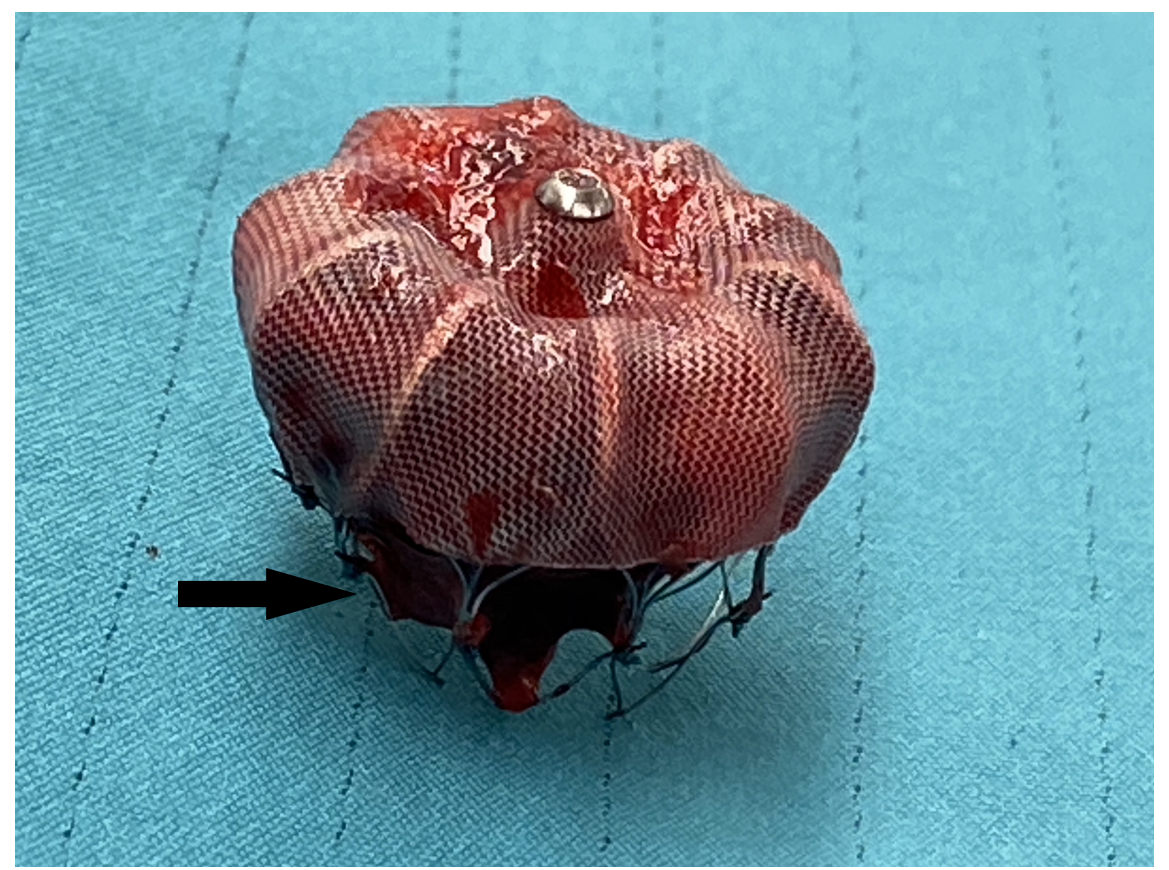

\section{Hosted file}

WatchmanInLAA .mp4 available at https ://authorea.com/users/316008/articles/476801-left-atrialappendage-perforation-by-the-watchman-device-considerations-for-emergency-repair

\section{Hosted file}

Watchman Removal.mp4 available at https://authorea.com/users/316008/articles/476801-leftatrial-appendage-perforation-by-the-watchman-device-considerations-for-emergency-repair 